\title{
«NATO ED ALLEVATO IN CORTE»: TORQUATO TASSO
}

\author{
Amedeo Quondam \\ (Sapienza Università di Roma) \\ amedeo.quondam@,uniroma1.it
}

\section{RIASSUNTO}

Con un'attenta analisi del dialogo Il Malpiglio overo della Corte il saggio descrive le continuità e le trasformazioni del discorso cortigiano attraverso il Cinquecento, da Castiglione a Tasso.

PAROLE CHIAVE: Torquato Tasso; dialogo rinascimentale; Corte, prudenza; onore.

\section{«NATO ED ALLEVATO IN CORTE»: TORQUATO TASSO}

\begin{abstract}
With a careful analysis of the dialogue Il Malpiglio overo della Corte, the essay describes the continuities and transformations of the courtier discourse through the sixteenth century, from Castiglione to Tasso.
\end{abstract}

KEY WORDS: Torquato Tasso; Renaissance dialogue; Court; prudence; honor.

Anche se deve avervi avuto una parte di rilievo l'incertezza che continua a segnarne la loro situazione propriamente testuale, dopo l'edizione critica che nel 1958 ne procurò Ezio Raimondi ${ }^{1}$, non saprei dire perché mai i venticinque dialoghi di Torquato Tasso non sembrano avere ancora incontrato un profondo e organico interesse negli studi, anche se non sono certo mancati pregevoli studi su singoli dialoghi, su gruppi di dialoghi e sui diversi problemi che questi testi propongono, in campo sia filologico sia interpretativo, anche rispetto alla grande tradizione dialogica, volgare e latina, umanistica e classicistica e alle stesse, talvolta assai tormentate,

\footnotetext{
1 Torquato Tasso, Dialoghi, ed. critica Ezio Raimondi (Firenze: Sansoni, 1958); l'avvertenza posta in frontespizio («edizione dei soli testi definitivi») riassume le difficoltà che la tradizione manoscritta e a stampa dei Dialoghi, così come tante altre opere tassiane, se non tutte, pongono a chi se ne volesse fare editore, con inevitabili ripercussioni sulla possibilità di affrontare in sicurezza l'analisi delle loro argomentazioni. Lo stesso Raimondi ha proposto una lettura complessiva dei dialoghi nell'introduzione alla loro nuova edizione completa a cura di Giovanni Baffetti (Milano: Rizzoli, 1998), 9-56. Il Malpiglio è stato pubblicato anche a cura di Bruno Basile nella raccolta di Dialoghi: Il Messaggiero, Il padre di famiglia, Il Malpiglio, La Cavalletta, Il Molza (Milano: Ugo Mursia Editore, 1991).
} 
contingenze biografiche della loro composizione ${ }^{2}$. Intendo dire che il corpus dialogico tassiano, più di quanto non sia stato finora fatto, può e deve essere considerato come un macrotesto, per quanto geneticamente disorganico e persino disordinato, anche perché la loro scrittura talvolta è dovuta a fortuite occasioni, in qualche caso strumentalmente funzionali alla ricerca di una personale sistemazione. Malgrado questi limiti, se considerati in quanto segmenti di un macrotesto, $i$ dialoghi rivelano le tante trame intertestuali che li innervano e li prospettano come una sorta di abbozzo per un'enciclopedia del Classicismo di fine Cinquecento, topicamente disposta per grandi temi: si potrebbe dire che i dialoghi di Tasso tendono a costituire una nuova Fabrica del mondo, ovviamente selettiva dei lemmi fondamentali nel lessico culturale che è proprio dei due decenni dell'esperienza dialogica tassiana, eppure in grado di cogliere gran parte di quelli che avranno un notevole futuro nella cultura barocca.

Scritti e riscritti nell'arco di quasi un ventennio (tra il 1578 e la morte del poeta nel 1595, in particolare nei sette anni del ricovero-prigionia in Sant'Anna a Ferrara: 1579-1586), ma con notevoli intervalli dipendenti anche dalle personali traversie di Tasso, i dialoghi affrontano infatti soltanto alcune grandi questioni contemporanee, ma dialogo dopo dialogo creano una fitta trama intertestuale che è in primo luogo semantica, oltre che senza precedenti né riscontri, per estensione e intensità, nell'intera tradizione classicistica. Al di là del loro tante contraddizioni e continue irresolutezze (tipicamente tassiane, si potrebbe dire, se non rischiasse di risolversi in un truismo) e al di là del loro sempre incerto destino editoriale (per lungo tempo sono stampati singolarmente), è questa macrotestualità dei dialoghi a risultare proiettata verso il futuro.

Basterà scorrerne i titoli, che in gran parte assumono il nome di uno dei loro interlocutori, per avere una prima, ma solida, impressione della notevole ampiezza

\footnotetext{
${ }^{2}$ Propongo solo alcuni studi recenti su alcuni aspetti dei Dialoghi in generale e su alcuni in particolare: Emilio Russo, "I dialoghi tassiani e la cultura di fine Cinquecento", in L'ordine, la fantasia e l'arte. Ricerche per un quinquennio tassiano (1588-1592) (Roma: Bulzoni Editore, 2002), 9-67; Franco Pignatti, "I Dialoghi di Torquato Tasso e la morfologia del dialogo cortigiano rinascimentale", Studi Tassiani 36 (1988): 7-43; Sergio Bozzola, “'Questo quasi arringo del ragionare'. La tecnica dei Dialoghi tassiani”, Italianistica 26, 2 (1997): 253-278; Matteo Residori, “Del fuggir la moltitudine'. Néoplatonisme et scepticisme dans le Malpiglio secondo du Tasse", Italique. Poésie italienne de la Renaissance 5 (2002): 95-108, https://doi.org/10.4000/italique.150; Angelo Chiarelli, "'Questa concordia è sempre ne le cose vere'. Note per una contestualizzazione de Il Costante overo de la clemenza di Tasso", Filologia \& Critica 16 (2016): 257-270, https://doi.org/10.1400/266543; Dante Fedele, "Uno scritto sull'ambasciatore del secondo Cinquecento: Il Messaggiero di Torquato Tasso", Il Pensiero Politico 51 (2018): 113-125; Giacomo Vagni, "Fra realtà biografica e verosimile letterario. Primi appunti sui personaggi dei Dialoghi di Tasso", in "Imitazione di ragionamento". Saggi sulla forma dialogica dal Quattro al Novecento, ed. Vincenzo Caputo (Milano: Franco Angeli, 2019), 127-138; Giacomo Vagni, “Oltre l'autocensura. Note sul rifacimento del dialogo tassiano Il Nifo overo del piacere", Rivista di letteratura italiana 37 (2019): 39-56, https://doi.org/10.19272/201902203002; sul Malpiglio: Virginia Cox, “Tasso’s Malpiglio overo de la Corte: The Courtier Revisited", The Modern Language Review 90, 4 (1995): 897-918, https://doi.org/10.2307/3733065; Massimo Lucarelli, "Il nuovo Libro del Cortegiano: una lettura del 'Malpiglio' di Tasso", Studi Tassiani 52 (2004): 9-24; Angelo Chiarelli, "Una 'congregazione di uomini raccolti per onore'. Tentativi di aggiornamento della teoria cortigiana nella dialogistica e nella prosa tassiana”, Rassegna della letteratura italiana 121, 1 (2017): 34-43.
} 
dell'orizzonte tematico dei Dialoghi e, al tempo stesso, della loro organica coerenza (li cito nell'ordine dell'edizione di Raimondi): Il Forno overo de la nobiltà, Il Beltramo overo de la cortesia, Il Forestiero Napolitano overo de la gelosia, Il N. overo de la pietà, Il Nifo overo del piacere, Il Messaggiero, Il padre di famiglia, De la dignità, Il Gonzaga secondo overo del ginoco, Il Rangone overo de la pace, Il Malpiglio overo de la Corte, Il Malpiglio secondo overo del fuggir la moltitudine, La Cavaletta overo de la poesia toscana, Il Gianluca overo de le maschere, Il Cataneo overo de gli idoli, Il Ghirlinzone overo l'epitafio, La Molza overo de l'amore, Il Costante overo de la clemenza, Il Cataneo overo de le conclusioni amorose, Il Manso overo de l'amicizia, Il Ficino overo de l'arte, Il Minturno overo de la bellezza, Il Porzio overo de le virtù, Il Conte overo de l'imprese, oltre a uno con il semplice titolo di Dialogo. Un insieme ragguardevole, che deve essere correlato alla sistemazione teorica che Tasso compie della forma dialogica nel breve Discorso dell'arte del dialogo, dedicato ad Angelo Grillo, elaborato nel 1585, nello stesso periodo in cui scrive il Malpiglio.

Si potrebbe disegnare una mappa semantica della trama continua e coerente dell'argomentazione dei Dialoghi, e avrebbe una forma ellittica, con due fuochi: da una parte avrebbe comunque e sempre Aristotele come suo referente obbligato, come suo collante universale, correlato a una padronanza generale della cultura classica che non ha riscontri tra i contemporanei: per quanto attiene la poetica, in primo luogo, e quindi l'estetica, ma anche la politica e l'economica, e soprattutto l'etica della Virtù e delle virtù; fino a ricadute tematiche molto settoriali, ma distintive delle dinamiche culturali di fine secolo e pur sempre variamente connesse con il paradigma aristotelico. L'altro fuoco dell'ellisse sarebbe quello della Corte: esplicitamente trattato (già nel titolo) nel dialogo che proporrò in questo mio intervento, Il Malpiglio overo de la Corte (edito nel 1587, ma scritto nel 1584-1585), connesso, però, in fittissima trama intertestuale (anzi, macrotestuale, come ho detto) non soltanto a molti degli altri dialoghi, se non a tutti, ma anche a tante lettere di questo periodo e alle altre opere tassiane ${ }^{3}$. All' Aminta, in modo particolare, scritta nel 1573 e pubblicata nel 1580, con un successo non solo editoriale poi travolgente: perché la sua favola pastorale, se instituisce drammaturgicamente un campo di radicale contrapposizione alla corte (ne è infatti protagonista l'altro dalla corte, il suo antagonista: il mito dell'Arcadia, elaborato già un secolo prima da Sannazaro), lo fa però performativamente sulla scena della stessa corte e per la corte (la prima rappresentazione fu infatti in quella di Ferrara, seguita da quella alla corte di Urbino). E questa ambigua contraddizione tassiana entrerà nei codici cortigiani delle culture di Antico regime, perennemente affascinate dai miti pastorali, in narrazioni e in immagini.

\footnotetext{
3 Posso soltanto qui ricordare le due lettere al pesarese Curzio Ardizio (attivo alla corte di Mantova come disegnatore e ritrattista) del 27 giugno 1584, la prima, e senza data la seconda (n. 290 e 291 dell'edizione Guasti: Torquato Tasso, Le lettere disposte per ordine di tempo, ed. Cesare Guasti, 5 voll. [Firenze: Le Monnier, 1852-1855], II, 278-288), che lo aveva invitato a «scrivere ['alcune stanze'] in biasimo della corte»: Tasso espone le ragioni del suo rifiuto e propone molti degli argomenti del Malpiglio, tanto che questa potrebbe essere stata l'occasione che ne sollecitò la stesura (rinvio a Maria Teresa Girardi, "Le lettere non 'poetiche' di Tasso come luogo di riflessione poetica", in Ricerche sulle lettere di Torquato Tasso, ed. Clizia Carminati ed Emilio Russo [Sarnico: Edizioni di Archilet, 2016], 25-43).
} 
Il primo riscontro della centralità della corte nel sistema dei Dialoghi, e più in generale delle opere di Tasso, è dato dalla fitta trama di occorrenze dello stesso lemma corte, e termini derivati e correlati (da cortigiano a cortesia e cortese, e altri): a una prima rilevazione ne sono risultate più di 200 per corte e più di 100 per le altre voci. Se poi si considerano, come è indispensabile, anche i dati di una biografia inquieta e mobile, e talvolta drammatica, alla perenne ricerca di una adeguata e conveniente sistemazione, è proprio la Corte a dominare la vita e la mente di Tasso. Le corti, anzi, al plurale, molto spesso nominate come luoghi della propria esperienza, in diverse sue opere, e in modo particolare nelle lettere, spesso scritte «dalla corte», da una delle tante in cui visse e variamente operò (per periodi più o meno lunghi: sempre nevrotici) o di cui ebbe modo di parlare. E furono corti di ogni tipo (regali, principesche, aristocratiche, ecclesiastiche: grandi e piccole) quelle in cui visse o fu accolto come ospite d'onore: «la corte di Ferrara», «la corte di Mantova», «la corte d'Urbino», «la corte de l'imperadore», «la corte cesarea», «la corte di Roma», «la gran corte in Francia». Negli impieghi della famiglia semantica di corte affiora talvolta anche la consapevolezza che non esiste soltanto questo mondo: «la città e la corte», «l'opinione de la corte o del mondo», «l'opinion del volgo e de la corte»; ma anche: la diversa «stima de gli onori e de le ricchezze che da' cortegiani e da' mercanti suol esserne fatta».

Ed è ancora la Corte a provocare, alla fine degli anni Settanta, quell'esplosione di psicosi paranoica di persecuzione che porterà Tasso alla lunga prigionia in una cella dell'ospedale di Sant'Anna a Ferrara: quando il disagio non più controllabile per una collocazione nel sistema della corte ferrarese, avvertita come inadeguata, si manifesta in modo drammatico come malattia mentale. Non sentirsi convenientemente considerato e trattato nel groviglio dei rapporti di corte non è affatto per Tasso un tema letterario, di antica fondazione e da sempre topico (dal De curialium miseriis è, tanto per fare un esempio, il titolo della famosa opera di Enea Silvio Piccolomini nel 1444), ma è vissuto come un'esperienza profondamente negativa che altera, e poi spezza per sempre, ogni equilibrio psichico e comportamentale, ogni forma di autocontrollo (indispensabile a corte più di ogni altra virtù: tra simulazione e dissimulazione), anche quando lo stato mentale e fisico di Tasso sembrerà migliorare negli ultimi anni di vita (ben pochi peraltro: neppure nove).

Considerazione ai margini, non marginale: dopo avere dominato l'immaginario letterario e figurativo dell'Europa di Antico regime, Tasso ne diventa un emblema intensamente anche sentimentale (nel senso di allora) quando l'Antico regime si dissolve: da Goethe a Byron il suo mito si rinnova e si attualizza proprio perché la sua esperienza umana e poetica è riconsiderata sotto il segno della malattia e della follia.

Nei limiti di questo mio intervento non potrò affrontare a tutto campo i tanti affioramenti, diretti e indiretti, nell'opera tassiana, del tema della Corte, anche nelle implicazioni personali ora accennate. Cercherò comunque, attraverso l'analisi del solo Malpiglio, di fornire qualche elemento almeno della trama macrotestuale e intertestuale costitutiva e propria dei Dialoghi tassiani, tenendo presente quanto profonda sia stata nell'esperienza non solo biografica di Tasso la consapevolezza di essere «nato ed allevato in corte» (come scrive nella famosa ed importantissima lettera a Orazio 
Capponi del luglio 1576 dove espone la favola della Gerusalemme liberata) ${ }^{4}$, e che da questo nascimento e dalla conseguente, appropriata, educazione, consegue anche la consapevolezza di non poter «essere senza ambizione», letteraria certamente, ma con legittime, nell'economia del proprio servizio cortigiano, aspettative di convenienti riconoscimenti.

Prima di entrare nel Malpiglio mi sembra opportuno ricordare in estrema sintesi alcuni dati essenziali della biografia tassiana per illustrare il senso e le proporzioni di quel «nato ed allevato in corte», proiettandolo nell'intero arco della sua vita di cortigiano (stabile od occasionale), mai tranquilla e sempre inquieta, talvolta nevroticamente frenetica. Sono queste, in estrema sintesi, le tappe principali della sua esperienza personale, di corte in corte: nasce l'11 marzo 1544 a Sorrento, dove qualche anno prima la famiglia si era trasferita da Salerno, ed è una nascita in corte, perché il padre Bernardo era dal 1532 segretario di Ferrante Sanseverino, che di Salerno era principe (fu poi costretto all'esilio e privato del titolo e dei beni quando nel 1551 entrò in conflitto con il viceré Pedro de Toledo e fu dichiarato ribelle); alla fin del 1556 Torquato raggiunge il padre che si era trasferito a Pesaro alla corte del duca Guidubaldo II Della Rovere; trascorre l'estate del 1564 a Mantova, dove il padre era diventato segretario del duca Guglielmo Gonzaga; nell'ottobre 1565 entra al servizio del cardinale Luigi d'Este, a Ferrara; nel gennaio 1572 diventa cortigiano stipendiato del duca Alfonso II d'Este; con le prime manifestazioni dei disturbi mentali fugge nel luglio del 1577 da Ferrara e dopo avere percorso freneticamente l'Italia, alla fine del 1578 ripara a Torino, accolto dal principe Carlo Emanuele e dal marchese Filippo d'Este; pochi mesi dopo, nel febbraio 1579, fugge anche da Torino e fa ritorno a Ferrara, dove i disturbi mentali esplodono, tanto che a marzo è rinchiuso in Sant'Anna, dove resta fino a luglio 1586; negli ultimi mesi del 1587 si stabilisce a Roma, ospite del cardinale Scipione Gonzaga, ma nel 1589 chiede a Ferdinando de' Medici, granduca di Toscana, di essere accolto al suo servizio; nell'aprile del 1590 è a Firenze in quella corte, ma a settembre è di nuovo a Roma; nei primi mesi del 1591 accetta l'invito di Vincenzo Gonzaga e torna a Mantova; nel febbraio 1592 è a Napoli, ospite prima del principe Matteo di Capua e poi del marchese Giovanni Battista Manso; torna a Roma a maggio, ospite del cardinale Cinzio Passeri Aldobrandini, nipote di Clemente VIII, e a Roma muore il 25 aprile 1595.

Uno dei documenti più drammaticamente intensi, e famosi, del rapporto di Tasso con la vita di corte è la lunghissima lettera scritta, nel settembre 1578, al duca di Urbino Francesco Maria della Rovere, di cui è ospite da qualche mese. La lettera, necessariamente e cortigianamente encomiastica nell'esordio e satura di dotti riferimenti ed exempla desunti dal repertorio classicistico, ma ricca anche di autodifese dalle tante malignità subite da parte di chi lo considera ormai segnato dalla pazzia, è la concitata narrazione dei tanti spostamenti di corte in corte che avevano segnato il periodo successivo alla fuga di Tasso da Ferrara (il 27 luglio 1577), che lo portò prima nella natia Sorrento, presso la sorella Cornelia, e poi (a fine anno) a Roma, e da qui, nell'aprile 1578, a Ferrara, per poi recarsi a Mantova, Padova, Venezia, e finalmente a

\footnotetext{
4 Tasso, Le lettere, I, 199 (lettera n. 82). 
Pesaro e Urbino, ospite del Duca, e da qui a settembre tornare ancora a Ferrara e poi a Mantova e infine approdare a Torino per entrare al servizio di Emanuele Filiberto, duca di Savoia. Sempre alla ricerca di una protezione che gli sembri appropriata, Tasso riversa nella lettera al Duca di Urbino un'angosciata e angosciante autocommiserazione, che è di per sé il segno più evidente di quanto profondo fosse il suo disagio mentale:

È certo miserabile cosa l'esser privo de la patria, spogliato de le fortune; l'andar errando con disagio e con pericolo; l'essere tradito da gli amici, offeso da' parenti, schernito da' servidori, abbandonato da' patroni; l'aver in un medesimo tempo il corpo infermo e l'animo travagliato da la dolorosa memoria de le cose passate, da la noia de le presenti, dal timor de le future: miserabile, che a la benivolenza si risponda con odio, a la simplicità con inganno, a la sincerità con fraude, a la generosità con bassezza d'animo: miserabile molto, ch'io sia odiato perch'io sia stato offeso; né sia ben voluto, perché dopo l'offese abbia amato gli offensori; ch'io perdoni a' fatti, altri non perdoni a' detti; ch'io dimentichi l'ingiurie ricevute, altri non dimentichi le fattemi; e ch'io desideri l'onor altrui ancora con alcun mio danno, altri desideri la mia vergogna senz'alcun suo pro5.

Per completare questo opportuno prologo su Tasso «nato ed allevato in corte» propongo ora qualche significativo riscontro microcitazionale dalle tante occorrenze del lemma corte nelle sue opere (soprattutto nelle lettere), iniziando da quelle che rinviano a esperienze personali, e dalle più brevi, con riferimenti generici: «essendo mio padre nella corte di Spagna per servizio del principe di Salerno suo padrone», «parlerò di questa materia come uomo di corte e di mondo», «io mi ritrovo in Turino in corte del signor marchese da Este», «vedete quanto obligo avrei a la corte o a le corti, perch'io scrivo a tutte o a molte, e da molte ho qualche risposta», «in corte, dove sono poi vissuto molti anni», «io non potrei sostener di vivere in corte con peggior condizione o con minor favore di quello c'ho avuto ne la mia gioventù», «in corte non può stare chi non dona almeno qualche scudo» ${ }^{6}$ E poi due citazioni più lunghe:

Chi vive in corte ha bisogno d'usare non solo prudenzia per sé, ma deve anche raccomandarla altrui per ciò che lo riguarda; perché questa in corte è più necessaria dei talenti, senza i quali può trovarsi favore e fortuna, ma senza quella non si trovano che disgrazie e malanni?

[...] io sono infermo senza dubbio, e così consumato ne gli studi; e nacqui gentiluomo, e vissi molt'anni in questa guisa: né potendo vivere ne la corte di Roma ne l'istesso modo, bisogna ch'io cerchi altro rifugio; peroché ogni diminuzione di favore o di grazia è una tacita licenza, o piuttosto una palese violenza ${ }^{8}$.

\footnotetext{
${ }^{5}$ Ibidem, I, 288.

${ }^{6}$ Per queste microcitazioni, come pure per le successive, mi limito a rinviare al corpus tassiano disponibile e interrogabile nel sito www.bibliotecaitaliana.it [data di consultazione: 10/2/2021].

${ }^{7}$ N. 1562, a Maurizio Cataneo, da Ferrara, 4 maggio 1572; v, 225-226.

${ }^{8}$ N. 1278, a Niccolò degli Oddi, da Roma, settembre 1590; v, 5-6.
} 
Altrettanto numerose sono le occorrenze del lemma cortigiano (circa 150) in tutti i suoi topici impieghi della tradizione discorsiva sulla Corte: sua propria condizione è la nobiltà, «la quale è l'antica ricchezza accompagnata da buoni costumi e da belle maniere» (così nel dialogo Il Forno, \ 74), in quanto «professione di cortegiano» (cioè, di "pratico cortigiano»: nella prassi ordinaria delle mansioni costitutive e proprie del suo prestare servizio per un signore che è suo padrone), magari di «buon cortegiano»; titolare di «prudenza cortigiana accoppiata con tanto ornamento di scelte e polite lettere e di felicissima eloquenza, quanto basta per farsi riconoscere per singolare» (così nel dialogo Il Messaggero, $\$ 202$ : a proposito di Battista Guarini). Sono numerosi anche $\mathrm{i}$ «cortegian malvagi», spinti da «invidia cortigiana» o da «l'invidia e la malivoglienza», che rendono infelice «la vita de' cortigiani», con quanto consegue nella ripresa del topico repertorio che da secoli - come ho ricordato - tratta de miseriis curialium, e in particolare della similitudine della Corte come insidioso mare con tanti pericolosi scogli (resa famosa dall'enunciazione della «regula universalissima» della grazia nel Cortegiano) ${ }^{9}$. Mi riferisco a quella topica che nel 1587, negli anni, cioè di Tasso trova della Filosofia cortesana moralizada di Alonso de Barros la sua risoluzione in gioco di società (il gioco dell'oca, ovvero la corte): ma citare quest'opera qui è come portare vasi a Samo ${ }^{10}$.

\section{Scrive Tasso nel dialogo Il Manso overo de l'amicizia:}

[...] la corte è simile al mare, in cui fa uopo esperto nocchiero; i cortigiani simili agli scogli coperti da l'onde che sogliono occultamente sommergere l'altrui fortune he sogliono occultamente sommergere l'altrui fortune; i venti contrari sono l'avversità di questo mondo; i mostri i vizî de gli infelici cortegiani, la cui virtù consiste ne lo schivargli $[\ldots](\$ 5)$.

E nell'ultimo dialogo, Il Conte overo de l'imprese, sviluppa ancora una similitudine marina, quella del nautilo, che un gentiluomo cortigiano ha voluto assumere come personale impresa, così significando che il nautilo è il cortigiano perché l'esperienza di entrambi è la tempesta, come esplicita il motto:

\footnotetext{
${ }^{9}$ La cito ancora una volta, tanto per promemoria: «Ma havendo io già più volte pensato meco onde nasca questa gratia, lasciando quegli che dalle stelle l'hanno, trovo una regula universalis-sima, la qual mi par valer circa questo, in tutte le cose humane che si facciano. E cioè, fuggir quanto più si pò, e come un asperissimo e pericoloso scoglio, la affettatione e, per dir forse una nova parola, usar in ogni cosa una certa sprezzatura che nasconda l'arte e dimostri ciò che si fa e dice venir fatto senza fatica e quasi senza pensarvi» (I, 4, 82-83); cito dall'edizione da me curata: Baldassare Castiglione, Il Libro del Cortegiano, ed. Amedeo Quondam (Roma: Bulzoni Editore, 2016).

${ }^{10}$ La citazione di questo "gioco dell'oca" intende essere un omaggio al caro amico José Martínez Millán, che è stato tra i primi a illustrarne l'importanza, e con lui a tutto lo IULCE; da parte mia ho dato un contributo nel saggio "Giochi di corte", in Giocare tra Medioevo ed età moderna. Modelli etici ed estetici per l'Europa, ed. Francesca Aceto e Francesco Lucioli (Roma: Fondazione Benetton Studi Ricerche-Viella, 2019), 87-112.
} 
Il nautilo non è il polpo, ma simile, come dice Aristotele, ne la forma de' capelli: ha la testa ne la schiena, esce da la profondità del mare, avendo la conca volta verso se medesimo per non prender acqua, e in questa maniera naviga, alzando a guisa di vela i due crini superiori, fra' quali è una membrana simile a quella de' piedi de l'anitre o d'altro uccello simigliante: gli altri due distende in mare in vece di timone; se vede cosa che gli venga incontra, raccoglie i piedi e, riempiendo la sua conca d'acqua, si sommerge nel profondo, dove suole ancora fuggir la tempesta. È impresa del signor Girolamo Catena, gentiluomo in questa corte di molte lettere e di molta esperienza e di molta reputazione, il quale ha voluto assomigliare la navigazione del nautilo a quella del cortegiano, e dichiara la sua intenzione con questo motto: TEMPESTATIS EXPERS $(\$ 188)$.

Di questa impresa propongo l'immagine tratta dal grande repertorio Teatro d'imprese di Giovanni Ferro, che, come fa per tutte, l'illustra ampiamente riorganizzando una tradizione discorsiva e iconica quasi secolare:

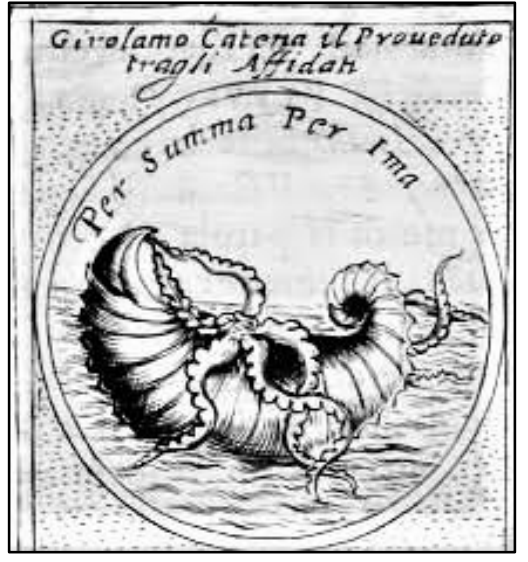

Fig. 1- Giovanni Ferro, Teatro d'imprese. 1623, Roma, Biblioteca Universitaria Alessandrina.

Prima di passare all'analisi del Malpiglio mi permetto di osservare ancora che il tema della Corte da sempre istituisce una rete semantica molto ricca e articolata, e soprattutto metamorficamente fluida nel tempo, ma in termini tali che la costituiscono in una delle invarianti macrostrutturali della tradizione discorsiva sull'etica propriamente pertinente alla forma del vivere; e osservo altresì che di tale rete semantica una delle articolazioni primarie è la cortesia, alla quale Tasso dedica un intero dialogo (Il Beltramo overo de la cortesia, in prima redazione nel 1579 ed edito nel 1586). Il lemma (e forme derivate) ha un cospicuo numero di occorrenze nel lessico tassiano (oltre 130), dalle quali risulta la correlazione genetica con la liberalità e con le altre virtù che ne conseguono, messa in evidenza nella tradizione non solo umanistica (in Pontano, a esempio). Devo però limitarmi a una sola citazione, e brevissima dal 
Beltramo, in cui è stata riconosciuto il rapporto diretto con il Convivio dantesco ${ }^{11}$, ed è questa: «[...] la cortesia è non una sola ma tutta la virtù di corte [...]» (\ 25), in quanto direttamente derivata dalla curialitas, mentre la liberalità è - come ho ricordato - una delle virtù che ne articolano le pratiche ordinarie nel sistema della corte ${ }^{12}$.

È a tre voci il dialogo Il Malpiglio: il gentiluomo di antica famiglia lucchese, Vincenzo Malpigli, da tempo alla corte estense ${ }^{13}$, chiede al Forestiero Napolitano (come sempre, maschera e portavoce dell'autore) di incontrare il figlio Giovanlorenzo, che desidera ragionare con lui «in qualche materia, e particolarmente de la corte» ( $\mathbb{S}$ $2)^{14}$. Il Forestiero Napolitano cerca subito di schermirsi, dichiarandosene poco esperto, ma Vincenzo Malpigli accortamente lo blandisce con obbligata captatio benevolentiae: «Ove manca per aventura l'esperienza, abonda [in voi] l'ingegno, il sapere e la dottrina, sì ch'a niun altro egli si potrebbe avvenire da cui più credesse d'intenderne» (\$2). Così sollecitato, il Forestiero Napolitano replica cercando ancora di sottrarsi al compito richiesto, esortando il giovane Malpigli a confrontarsi con chi di corte è esperto non per prattica ma per scienza e intanto a leggere, nella bibliografia disponibile (diremmo oggi), quei libri che non ne trattino, di nuovo, solo per prattica, ma con l'obiettivo di perseguire la formazione dell'idea del perfetto cortigiano:

S'egli non cerca i prattici cortigiani, ma coloro che ne parlano o scrivono per alcuna scienza, molti potrà ritrovarne, a' quali io sono tanto inferior di sapere quanto minor d'età; ma fratanto può leggere i libri di coloro c'hanno formata l'idea del cortigiano $(\mathbb{S}$ 3).

Per vincere le residue resistenze del Forestiero Napolitano, Vincenzo Malpigli gli certifica che suo figlio ha già fatto buone letture (una soprattutto: «[...] ha letto il Cortigiano del Castiglione e lo ha quasi a mente, e forse meglio che l'Epistole di Cicerone o le Comedie di Terenzio [...]»), ma che ha un problema: avendo appreso che

${ }^{11}$ Fiammetta Papi, "Sulla semantica della cortesia. Riflessioni su una definizione dantesca", Italianistica. Rivista di letteratura italiana 44, 2 (2015): 209-221 (per Tasso: 218-221), https://doi.org/10.1400/235710.

12 Per una più estesa trattazione di questi temi mi permetto di rinviare al mio studio Forma del vivere. L'etica del gentiluomo e i moralisti italiani (Bologna: Il Mulino, 2010).

${ }^{13} \mathrm{Dal} 1578$ era a Ferrara, prima impegnato in bonifiche del territorio paludoso, poi come tesoriere del duca Alfonso II; figlio di Nicolao Montecatini e di una figlia di Giovan Lorenzo Malpigli era stato adottato dal nonno materno e ne aveva ereditato nel $1541 \mathrm{i}$ beni e il cognome. Le notizie su di lui dipendono dalla tragica fama della figlia Lucrezia, complice dell'omicidio del proprio marito (le era stato imposto dalla famiglia), Lelio Buonvisi, e, riparata in convento dopo l'omicidio, di scandalose storie di amori (in queste vicende fu sostenuta anche economicamente dal fratello Giovanlorenzo; rinvio a Salvatore Bongi, Storia di Lucrezia Buonvisi lucchese raccontata sui documenti [Lucca: B. Canovetti, 1864; Lucca: Pacini Fazzi, 1978, ristampa anastatica], e alla voce "Lucrezia Malpigli" di Carla Sodini nel Diqionario Biografico degli Italiani, LXVIII [Roma: Istituto della Enciclopedia Italiana, 2007], ad vocem, disponibile in rete sul sito www.treccani.it [data di consultazione: 10/2/2021]).

${ }_{14}$ Tasso fu in rapporto soprattutto con Giovanlorenzo, che è interlocutore ancora del Forestiero Napolitano nel dialogo a due voci Il Malpiglio secondo overo del fuggir la moltitudine, scritto tra il 1584 e il 1585, insieme al dialogo sulla Corte: restano alcune lettere tassiane a lui indirizzate nel 1586 (e una a suo padre), e del secondo dialogo parla in alcune altre. 
«le corti si mutano nel tempo» (fondamentale enunciato da cui muove l'argomentazione dello stesso Cortegiano) vorrebbe «intender cose nuove» (\$3).

A questo punto il Forestiero Napolitano non può che accettare la sollecitazione di Vincenzo Malpigli. Decide però di inaugurare la sua partecipazione al dialogo con la famosa apologia del libro di Castiglione ${ }^{15}$, attestando così come e quanto, ancora a fine secolo, questo libro resti l'obbligato punto di partenza di ogni discorso sulla corte, e continui ad avere questa funzione proprio perché - secondo Tasso che impiega le categorie su cui a lungo ha riflettuto, tra Platone e Aristotele - Castiglione ha formato l'idea del perfetto cortigiano, stabile e ferma in quanto tale, non una sua effimera imagine sottoposta alle mutazioni dei tempi:

Chi forma l'idea non figura alcuna imagine che si muti con la mutazione fatta de gli anni, ma, isguardando in cosa stabile e ferma, la ci reca ne' suoi scritti quale nel pensiero l'ha formata. Né stimo già che 'l Castiglione volesse scrivere a gli uomini de' suoi tempi solamente, tuttoch'egli alcuna volta faccia per gioco menzione di que' più vecchi cortigiani i quali al tempo di Borso portarono lo sparaviero in pugno per una leggiadra usanza: perché la bellezza de' suoi scritti merita che da tutte l'età sia letta e da tutte lodata; e mentre dureranno le corti, mentre i principi, le donne e i cavalieri insieme si raccoglieranno, mentre valore e cortesia avranno albergo ne gli animi nostri, sarà in pregio il nome del Castiglione $(\$ 4)^{16}$.

Nel lessico tassiano (in rapporto non solo con Platone) l'idea, ogni idea, è oltre la natura e il tempo (storia compresa), con le tante loro diverse occasioni, e pertanto non subisce le mutazioni, che possono tutt'al più incidere sui dettagli: «Ma s'alcuna cosa è forse la qual si cambi e si vari co' secoli e con l'occasioni, non è di quelle che son principali nel cortigiano [...]», e dunque ha fatto bene il giovane Malpigli a scegliere come «suo famigliare il formator de le corti», cioè il libro di Castiglione, e non «lo scrittor de le comedie», cioè Terenzio ( $\int 5$; nulla, però, è detto ora a proposito di

${ }^{15}$ Converrà ricordare che Tasso aveva composto un sonetto in lode di Baldassarre Castiglione (è il n. 1517 delle Rime) per l'edizione del libro di Pietro Grizi, Il Castiglione overo dell'arme di nobiltà, che era stato pubblicato da Antonio Beffa Negrini nel 1586 (a Mantova, edito da Francesco Osanna), molto legato al conte Camillo Castiglione, figlio primogenito di Baldassarre; Tasso aveva ricevuto il libro mentre era a Mantova e scrisse a Beffa Negrini per ringraziarlo, citando anche il conte Camillo: ne è traccia il biglietto ad Ascanio Mori (Tasso, Le lettere, n. 678, III, 75-76; fu scritto nel novembre di quell'anno); a Beffa Negrini scrive ancora il 29 giugno 1591, da Mantova, inviandogli di nuovo quel sonetto (e altri due) per l'edizione dei suoi Elogi historici di alcuni personaggi della famiglia Castigliona (saranno stampati postumi nel 1606, ancora a Mantova da Osanna), di nuovo citando il conte Camillo; altri biglietti di Tasso a Beffa Negrini: n. 537 (II, 563, senza data), n. 675 (III, 3, del novembre 1586), n. 677 (III, 74-75, nello stesso mese), n. 689 (III, 84, del 22 novembre 1596), n. 712 (III, 103, del dicembre 1586), n. 754 (III, 148, del gennaio 1587); Beffa Negrini è citato anche nella lettera n. 634 (III, 30, dell'agosto 1586).

${ }^{16}$ L'elogio di Castiglione ha una ulteriore conferma nelle battute successive, quando Malpigli padre commenta le parole del Forestiero Napolitano («Se per l'adietro egli volentieri leggeva il Cortigiano, per l'avvenire no 'l lascerà giamai, poiché da voi tanto è commendato, al quale non soglion piacere tutte le cose che piacciono a gli altri»), che risponde dichiarando il proposito di tornare sul libro di Castiglione: «Molte sono le cagioni per le quali onoro la memoria del Castiglione, e mi riserbo di parlarne con maggiore opportunità» (\ 6$)$. 
Cicerone). Non mi addentro oltre nell'analisi degli impieghi tassiani di idea, fondamentali nei Discorsi del poema eroico, e non solo ${ }^{17}$, e dunque mi limito a rilevare che le occorrenze di maggiore intensità semantica di questo lemma nel lessico tassiano sono correlate al verbo formare (l'idea in quanto principio genetico della forma) e quindi alla perfezione: con un implicito ma costante rinvio all'impostazione argomentativa di Castiglione, tradizionalmente ( $\mathrm{da}$ Ariosto in poi, fino a questo dialogo) definito come il formatore del cortigiano, ma in quanto perfetto cortigiano ${ }^{18}$. Il che del resto corrisponde alla stessa consapevolezza di Castiglione, che nella dedica interna ad Alfonso Ariosto del primo libro del Cortegiano aveva scritto:

Voi adunque mi richiedete ch'io scriva qual sia al parer mio la forma di cortegiania più conveniente a gentilhomo che viva in corte de principi, per la quale egli possa e sappia perfettamente loro servir in ogni cosa ragionevole, acquistandone da essi gratia e dagli altri laude. Insomma, di che sorte debba esser colui che meriti chiamarsi perfetto cortegiano, tanto che cosa alcuna non gli manchi $(\mathrm{I}, 1,3)$.

Ed è certo ridondante ricordare che anche il gioco scelto dai cortigiani riuniti nel Palazzo ducale di Urbino era stato quello di «formar con parole un perfetto cortegiano» ${ }^{19}$.

${ }^{17}$ A esempio, è diretta la ripresa del Cortegiano nell’ Apologia: «Assai lodevolmente formò Senofonte l'idea del perfetto principe [...]»; e analogamente nei Discorsi: «[...] chi vuol formare l'idea d'un perfetto cavaliere [...]»; e ancora, alcune microcitazioni per documentare l'universale efficacia operativa dell'idea, sia nei campi dell'etica sia in quelli delle «cose artificiali»: «si forma l'idea della bontà e dell'eccellenza», «l'idea del poema eroico», «l'idea è 'l vero essemplare e 'l vero esempio» ed «[è] nel primo grado della verità», «il fine del poeta [è] riguardare nell'idea del bello», «l'idee son quasi germe e [...] sono avanti a tutte le cose», «l'idea del bello», «[l']idea della bellezza», «l'idea [...] del perfettissimo poema», «['] idea del bene», «l'idea universale del mondo», «l'idee son quasi germe», «l'idea del perfetto ambasciatore», «l'imagine sarà più bella quanto fie più simile a l'idea», «l'idee de la republica», «l'idee sono cause universali»; e per la sostanziale equivalenza tra forma e idea: «come fanno que' pittori, la cui laude principale consiste ne la forma o ne l'idea», «concedasi dunque che siano l'idee e le forme quasi disegni o modelli de le fabriche», «ma forme sono quell'altre ch'idee son state chiamate» (anche per queste per microcitazioni di idea rinvio al corpus tassiano disponibile nel sito di "Biblioteca Italiana": vi ho rilevato oltre 130 occorrenze del lemma).

${ }^{18}$ Nei termini che Castiglione stesso si era attribuito nella Dedica del libro, dove aveva replicato a chi l'aveva criticato ritenendo che fosse «tanto difficile e quasi impossibile trovar un homo così perfetto come io voglio che sia il cortegiano»: «A questi rispondo che mi contentarò haver errato con Platone, Xenophonte e Marco Tullio, lassando il disputare del mondo intelligibile e delle idee, tra le quali, sì come (secondo quella opinione) è la idea della perfetta republica e del perfetto re e del perfetto oratore, così è anchora quella del perfetto cortegiano: alla imagine della quale s’io non ho potuto approssimarmi col stile, tanto minor fatica haveranno i cortegiani d'approssimarsi con l'opere al termine e meta ch'io col scrivere ho loro proposto» (Dedica 33). La stessa impostazione torna nella dedica del primo libro ad Alfonso Ariosto, dove forma sta propriamente per idea: «Voi adunque mi richiedete ch'io scriva qual sia al parer mio la forma di cortegiania più conveniente a gentilhomo che viva in corte de principi, per la quale egli possa e sappia perfettamente loro servir in ogni cosa ragionevole, acquistandone da essi gratia e dagli altri laude. Insomma, di che sorte debba esser colui che meriti chiamarsi perfetto cortegiano, tanto che cosa alcuna non gli manchi» (I, 1, 3).

${ }^{19}$ Il contesto di questa famosa sintesi dei dialoghi urbinati è così formulato: «Per reprimere adunque molti sciocchi, i quali, per esser prosuntuosi ed inepti, si credono acquistar nome di bon cortegiano, 
Tornando al Malpiglio, esauriti i convenevoli di apertura e chiarite le esigenze dei Malpigli, il Forestiero Napolitano propone preliminarmente una precisazione in merito alla forma del suo consueto dialogare, fatto di «parole assai popolari, a le quali ne son mescolate alcune raccolte da' libri, non per istudio posto da me nel parlare, ma per usanza ch'io ho di leggere o di scrivere: e per questa cagione non ragiono se non famigliarmente con gli amici co' quali ho ragionato altre volte in questo soggetto» ( $\mathbb{(}$ 7). Esattamente nei termini in cui si erano compiuti i ragionamenti "famigliari" che avevano scandito il gioco del cortigiano in Palazzo ducale a Urbino (nel libro di Castiglione, ovviamente), sia la «privata e famigliar conversazione» tra due amici che si era svolta qualche anno prima del Malpiglio a Casale (nella Civil conversazione di Stefano Guazzo; princeps: 1574).

Con queste premesse il dialogo tra il Forestiero Napolitano e il giovane Malpigli può iniziare. Il problema sul quale Giovanlorenzo chiede lumi è però solo in parte quello di Castiglione, perché nel corso di mezzo secolo il discorso cortigiano ha messo in rilievo gli aspetti negativi della vita in corte, accentuando quella topica di cui ho detto, e allargando l'analisi non solo ai rapporti tra il cortigiano e il suo principe, ma anche a quelli del cortigiano con gli altri cortigiani; ed è qui il problema di Giovanlorenzo: «Io vorrei spezialmente sapere come s'acquisti la grazia de' principi e come si schivi l'invidia e la malivoglienza de' cortigiani» (\$ 11). È questo infatti il conflitto, endemico a corte, che ne connota le dinamiche relazionali, al tempo stesso positive e negative: «[...] perché ne la grazia del principe e ne la benevoglienza de i cortigiani tutte l'altre cose paiono esser contenute». Per poter affrontare tale problema il Forestiero Napolitano deve sapere quale sia lo scopo della richiesta del giovane Malpigli («Ma questo a che fine, ragionarne solamente o pur d'operare?» [ 11$]$ ); e la risposta è chiara:

In vero non mi spiacerebbe l'esser cortigiano, perch'io sono allevato in questa città [Ferrara], ne la quale il valor de gli uomini risplende più chiaramente ne le corti ch'in altro luogo; ma nondimeno mi sarebbe grave di tralasciare gli studî, perché mi pare che ne le corti simili a questa accrescano molto d'ornamento a' cavalieri ( $\$ 12)$.

Di rincalzo Malpigli padre osserva: «[...] de la cavalleria s'è invaghito parimente». Anche questo ulteriore allargamento dell'orizzonte argomentativo ribadisce l'impianto del Cortegiano, che tanta parte aveva dedicato proprio alla necessaria integrazione, nel cortigiano, tra le armi, nelle loro diverse operazioni, in pace e in guerra, e le lettere: in quanto cavaliere di nobile nascita, e dunque per principale professione dedito al mestiere delle armi, il cavaliere cortigiano avrebbe dovuto - secondo Castiglione - persuadersi che il «supremo ornamento» della propria distintiva condizione fossero le lettere.

vorrei che 'l gioco di questa sera fosse tale che si elegesse uno della compagnia ed a questo si desse carico di formar con parole un perfetto cortegiano, esplicando tutte le conditioni e particular qualità che si richieggono a chi merita questo nome $[\ldots] \gg(\mathrm{I}, 3,45)$. 
Messo secolo dopo, questo è un ormai un consolidato paradigma e pertanto il dialogo può acquistare velocità: prima riassume le ordinarie «operazioni del cavaliero» a corte (cioè, gli «esercizi del corpo», che tanto spazio avevano invece nel fondativo Cortegiano), per passare a ragionare del «valor de l'animo» e alle «virtù de' costumi» ( $\mathbb{\Omega}$ 14), a quelle virtù, cioè, che sono ora più apprezzate in corte. Il Forestiero Napolitano espone pertanto al giovane Malpigli una scorciatissima, ma precisa, sintesi della tradizione umanistica dell'institutio della nobile gioventù, quella tradizione che proprio negli anni in cui Tasso attende al Malpiglio, sta per essere riorganizzata e rilanciata dalla ratio studiorum dei gesuiti:

Dunque si debbono apprender le matematiche scienze e la filosofia de' costumi ['etica] e la naturale e la divina, e aver buona cognizione de gli istorici e de' poeti e de gli oratori e de l'arti più nobili, come sono quella de lo scolpire e del pingere e l'architettura: e di tutte queste cose il cortigiano dee tanto sapere che non possa alcuno riprenderlo d'ignoranza, perch'in tal guisa egli sarà molto onorato dal principe, e la benevolenza seguirà l'onore $(\mathbb{1 6})$.

È l'acquisizione di questa «eccellenza di tutte queste arti» a produrre l'invidia dei cortigiani che non hanno voluto o saputo raggiungerla con lo studio e la fatica, e pertanto - conclude il Forestiero Napolitano, andando ben oltre il perimetro del Cortegiano - è questa la contraddizione strutturale della vita di corte, la sua genetica ambiguità e la sua difficoltà, che la trasformano in quel pelago dove è molto pericoloso navigare, tanto da rendere tempestatis expers il cortigiano avveduto e rendergli impossibile «esser felice»:

Quelle cose medesime dunque le quali acquistan la benevoglienza de' principi, generan l'invidia cortigiana: laonde, non si potendo l'una e l'altra conseguire, non ci debbiamo curar d'esser invidiati da la corte, o non conviene con tanto studio ricercar la grazia de' signori $(\$ 17)^{20}$.

Il momento centrale del dialogo è quando il Forestiero Napolitano rivolge, con il solito metodo socratico, questa domanda al giovane Malpigli: «[...] non vi pare che la corte sia un'adunanza overo una compagnia?» (\$19). Per poi distinguere le adunanze tra quelle fatte per diletto (con rinvio esemplare al carnevale) e quelle fatte per utilità («come le compagnie di mercanti»), ma la corte, «quantunque ad alcuni sia molto utile,

${ }^{20}$ Richiederebbe un'analisi approfondita l'osservazione del Forestiero Napolitano che consegue da questa difficoltà, perché richiama la categoria la categoria della "disgiunzione", che Tasso stesso riconosce come imperfezione distintiva del proprio stile, perché la usa troppo frequentemente, in una famosa lettera al cardinale Scipione Gonzaga del $1^{\circ}$ ottobre 1575 (Tasso, Le lettere, I, 115 [lettera n. 47]): in quanto "parlar disgiunto», definito come «quello che si lega più tosto per l'unione e dependenza de' sensi, che per copula o altra congiunzione di parole», e che è consapevole adattamento della dissoluta locutio elaborata dalla retorica classica, praticato da Tasso per dichiarata imitazione di Virgilio. L'osservazione del Forestiero è questa: «Dunque per altre vie che per queste di tante virtù, di tante scienze e di tante cose apparenti e risguardevoli dee procedere il cortigiano a due fini così disgiunti, se pur le cose disgiunte si possono congiungere per artificio» ( $\left.\int 18\right)$. 
a molti piacevole», «non è congregata per utile o per diletto simplicemente», ma per onore, che altro non è se non il «servizio del principe» (\$ 20-21). La conclusione di questo ragionamento è semplice e chiara e segna una profonda mutazione rispetto al Cortegiano: evidenzia infatti quella marcatura semantica della categoria dell'onore destinata a diventare emblema distintivo della nuova cultura aristocratica già nell'età barocca, sempre più remota dai fondativi valori cortesi della cavalleria e del miles litteratus e sempre più cortigiana: «La corte dunque è congregazion d'uomini raccolti per onore» $(\mathbb{S} 21)^{21}$.

Su questo primario assioma il Forestiero Napolitano elabora una sottile analisi della differenza tra questo onore cortigiano e quello che «s'acquista ne le republiche ancora» (\$ 22), per evitare che il giovane Malpigli possa erroneamente pensare che «se la republica e la corte sono l'istessa adunanza, l'onore il quale si propone per fine dovrebbe esser il medesimo», mentre invece «se le compagnie son diverse, diverso parimente sarà l'onore», che è poi l'obiettivo di tutto il suo ragionamento. L'andamento dell'argomentazione del Forestiero Napolitano assume movenze sillogistiche, tipicamente aristoteliche: «[...] concedendo quello che si conosce chiaramente, la republica non esser corte, mi concederete che non sia l'istesso onore quel che ne l'una e ne l'altra è ricercato [...]», e dal momento che il giovane Malpigli desidera gli onori della corte e non quelli della repubblica, non deve fare l'errore di prendere «gli uni per gli altrì ( $(23)$, dal momento che sono e sembrano tra loro simili, ma in realtà sono molto diversi.

La necessità di tenere ben distinte, affinché «la somiglianza non c’inganni» ( $\mathbb{S}$ 24), queste diverse economie, non solo simboliche, dell'onore, quella del cittadino di repubblica e quella del cortigiano al servizio del principe, rende molto serrata questa parte del dialogo, che è propriamente "politica": i cittadini perseguono gli onori «maggiori e supremi» della repubblica perché «il desiderar sovrano onore ne la republica altro non è che desiderio di commandare» (\$25). Il giovane Malpigli, forse memore delle radici lucchesi della sua famiglia e degli ordinamenti repubblicani di quella città, interviene a questo punto con un'osservazione importante, che mette in gioco la funzione positiva delle «buone leggi» che regolino e temperino il «desiderio di comandare» da parte di «uomini che son cresciuti in libertà» ed evitino il rischio di tiranni e usurpazioni. La replica del Forestiero Napolitano, improntata ovviamente a grande cortesia, ma molto abile nel proporre positivamente l'esperienza di quelle repubbliche che «mutino forma in meglio» affidandosi a «principi prudentissimi», con un'allegazione esemplare che resta in sospeso (splendido dettaglio di sapienza dialogica):

Né io altramente intendo, quantunque molte volte le republiche mutino forma in meglio, e si conceda per utilità publica autorità sovrana a principi prudentissimi, come fu...; la quale autorità molti hanno cercata, molti non rifiutata, adoprandola per beneficio di coloro a' quali si commanda ( $\$ 26)$.

${ }^{21}$ Sul tema dell'onore come centrale nella nuova cultura cortigiana insiste opportunamente Chiarelli nel saggio citato; sull'onore restano comunque fondamentali le osservazioni di Carlo Dionisotti in Geografia e storia della letteratura italiana (Torino: Einaudi, 1967). 
Subito dopo il Forestiero Napolitano insiste sul significato di questa mutazione, che considera politicamente esemplare dei tempi nuovi, e la riferisce in modo diretto ed esplicito al caso del giovane Malpigli: benché «figliuolo di tanti illustri cittadini, i quali han commandato a gli altri leggitimamente» (cioè, in una bene ordinata repubblica), vuole interrompere questa tradizione perché ha maturato un diverso «desiderio d'onore»: servire a corte e non commandare i suoi concittadini. Questa radicale mutazione (dalla libertà alla servitù) è possibile perché Malpigli è spinto dallo «splendor d'alcuna rara virtù», distintiva e propria di chi sia di «animo generoso» (in quanto nobile): in questo modo, sotto il segno della virtù, Tasso proietta la scelta personale di Malpigli come rappresentativa dei processi generali che hanno rapidamente trasformato le prime corti domestiche e consortili in corti signorili, fondative di modelli politici e culturali, e quindi nelle moderne corti, sempre più burocratiche e rituali ${ }^{22}$. Secondo Tasso si è trattato, e si tratta, di processi che hanno riguardato, e riguardano, in primo luogo la Virtù e le virtù, cioè la sfera dell'etica come universale ordinatrice e regolatrice della politica (e dell'economica), oltre che dell'estetica: un'etica sostanzialmente aristotelica, insomma.

È questo il nuovo snodo argomentativo del dialogo: alla domanda del Forestiero Napolitano, proposta, come sempre, con socratica funzione maieutica («quale stimate voi che sia la virtù che si ricerca principalmente al buon cittadino?» [S 32]), Malpiglio risponde indicando la fortezza e la liberalità, che nelle repubbliche «son tanto onorate, come testimoniano le statue dirizzate a' valorosi, l'orazioni funebri e i versi e gli altri segni d'onore publici e privati». E quando il Forestiero Napolitano lo incalza chiedendogli se la fortezza sia virtù primaria anche per il cortigiano, non può non riconoscere che lo sia, perché il cortigiano è un nobile cavaliero, al quale «più si conviene il saper adoperar l'armi per onor proprio e per servigio del suo principe» ( $\mathbb{S}$ 33), così favorendo la replica del suo abile interlocutore che vuole portarlo a conoscere e riconoscere la differenza costitutiva e propria dell'onore cortigiano: la fortezza, «così civile come cortigiana», può essere una pratica rischiosa se esercitata senza guida o freno, o senza «chi la regga e indrizzi», cioè senza la virtù della prudenza.

A proposito di questa fase del dialogo mi sembra opportuno osservare che nella propria argomentazione il Forestiero Napolitano utilizza non solo alcuni exempla di protagonisti (negativi) della storia romana, capaci di fortezza priva però dell'indispensabile prudenza, ma anche una topica similitudine derivata dal mondo del cavallo («La fortezza a me par simile a’ destrieri generosi, che quanto sono più feroci, tanto hanno maggior bisogno di morso», cioè di quel freno già presente a testo $\int 34$ ). Questo dettaglio lessicale richiederebbe una digressione sulle altre parole che l'addestramento del cavallo (in quanto anch'essa vera e propria forma di institutio, con

22 Per il piacere di ricordare esperienze ormai remote mi piace continuare a usare queste definizioni, che restano significative dei processi di mutazioni del sistema delle corti, anche se superate dagli studi più recenti, e che furono proposte da Marco Cattini e Marzio A. Romani nella primissima fase di avvio delle ricerche di Europa delle Corti, nel loro importante saggio "Le corti parallele: per una tipologia delle corti padane dal XIII al XVI secolo", in La Corte e lo spazio: Ferrara estense, ed. Giuseppe Papagno e Amedeo Quondam (Roma: Bulzoni Editore, 1982), 47-82. 
le sue distintive pratiche e strumenti per il suo buon "reggimento") ha fornito diverse all'etica moderna della virtù, al suo lessico di base nelle sue invarianti micro e macrostrutturali ${ }^{23}$. Sulla base di queste considerazioni ed exempla il Forestiero pone al giovane Malpiglio la domanda decisiva nella sua strategia argomentativa («chi pare a voi più nobile, il cavallo o 'l cavaliero, il guidato o la guida, lo sfrenato o chi pone il freno?» \35), perché la orienta Malpigli a riconoscere la «maggior nobiltà» di chi ha la funzione di guida e di freno, tramite la prudenza, che «è scorta de la fortezza», è pertanto è la «più nobil virtù: e questa nel cittadino è civile e nel cortigiano peraventura è cortigiana prudenza». Come sempre, nelle argomentazioni dell'etica classica e classicistica, a fare la differenza è il diverso fine perseguito da queste diverse prudenze, che il Forestiero Napolitano risolve prevedibilmente in questo modo, perché è questo il fine che persegue: «percioch'il cortigiano ha per fine la riputazione e l'onor del principe, dal qual si deriva il proprio come rivo da fonte; e 'l cittadino la conservazione de la libertà» (\$ 36).

La prudenza repubblicana a questo punto scompare e il dialogo è tutto rivolto alla prudenza cortigiana, che è in primo luogo quella del principe, in quanto «architetto per rispetto [nei confronti] de gli operari» $(\S 38)$, cosicché la prudenza del cortigiano «consisterà ne l'essercitare i commandamenti del principe». Questa pratica dell'obbedienza è in realtà un impegno quotidiano pieno di difficoltà e d'insidie, perché riguarda il rapporto diretto, frontale, tra principe-padrone e cortigiano-servitore, un rapporto che già gli umanisti avevano analizzato (Giovanni Gioviano Pontano in primis, con il suo trattato De obedientia, scritto nel 1470-1472), cosicché a fine Cinquecento può essere delineato da Tasso attraverso le parole del Forestiero Napolitano, che consentono però di misurare quanto sia mutata la condizione del servizio a corte rispetto a quanto Castiglione aveva scritto a proposito del rapporto tra il cortigiano e il suo principe, in particolare nel quarto libro:

Ma l'essecutore e 'l ministro, in quanto egli è tale, è sempre inferiore a colui che gli commanda. Dunque dee il cortigiano in guisa operare ciò che gli è imposto che dimostri prudenza inferiore non sol di persona inferiore: e molte volte è disdicevole ch'egli spii le cagioni di quel che gli è commandato, o che voglia più saper di quel che gli conviene; ma con la sua piacevolezza e con la destrezza modera la severità de le commissioni, e come i venti prendon qualità da' luoghi onde passano, divenendo tepidi per camino, così le severe commissioni per l'accortezza del cortigiano sogliono parer men dure e spiacevoli il più de le volte (\$39).

Questa è dunque la parte che resta al cortigiano che serve il suo signore, in quanto essecutore e ministro dei suoi commandamenti: stare al suo posto, che è di «persona

${ }_{23}$ Anche in questo caso mi piace rinviare (oltre che al mio già citato Forma del vivere) a un libro che continuo a ritenere molto importante: Pierangelo Schiera, Specchi della politica. Disciplina, melancolia, socialità nell'Occidente moderno (Bologna: Il Mulino, 1999), 185-232; per la tradizione discorsiva sul cavallo mi permetto di rinviare anche al mio saggio "La gloria del cavallo", in Movilidad cortesana y distinción: coches, tiros y caballos. II Congreso Internacional "Las caballerizas reales y el mundo del caballo", ed. Juan Aranda Doncel y José Martínez Millán (Córdoba: IULCE-Córdoba Ecuestre, 2019), 13-44. 
inferiore», e dunque dotato di prudenza inferiore, ma praticando l'obbedienza con le virtù esteriori e performative della piacevolezza e della destrezza: facendosi accorto, come i venti. Questa similitudine tassiana non ha bisogno di commenti, nella sua nitidezza, tanto disincantata quanto brutale: sulla scena della Corte conta sempre più ormai il parere e non l'essere. Il Forestiero Napolitano insiste su questo tema: il cortigiano deve imparare "più tosto d'occultare che di apparere» (\$ 41), e a Malpigli che ritiene difficile «celar quel ch'io sono», precisa quali debbano essere i modi avveduti di questa pratica del nascondimento e della dissimulazione perché possa risultare virtuosa e considerata in modo positivo a corte:

Questo nascondersi nondimeno si può fare con alcuno avvedimento, per lo quale la picciola parte che si dimostri generi desiderio di quella che si ricopre, e una certa stima e opinion de gli uomini e del principe medesimo, che dentro si nasconda un non so che di raro e di singolare e di perfetto $[\ldots](\$ 42)^{24}$.

Ne consegue uno spunto argomentativo che Tasso non sviluppa in modo adeguato alla sua rilevanza futura nelle società di corte: prospetta infatti una differenza di comportamenti, nell'apparire e nell'essere, tra coloro che sono «amatori del principato» e coloro che amano la persona del principe, rafforzata dalle due immediate allegazioni esemplari proposte da Malpigli (dimostra così di avere fatto buoni studi), di due protagonisti del mito di Alessandro Magno: Efestione, amatore della persona di Alessandro Magno, e Parmenione, «amatore del principato» (era stato già generale di Filippo di Macedonia), finito in disgrazia e condannato a morte per la congiura contro Alessandro tramata da suo figlio Filota. Con entusiasmo il giovane Malpigli dice: «Io amerei meglio essere un giorno simile ad Efestione che molti anni eguale a Parmenione: laonde niun mio difetto mi curerei di celare al principe, sì veramente ch'egli insieme conoscesse la fede». Ma il saggio Forestiero Napolitano ragiona a fondo sugli exempla antichi, utilizzandoli come prototipi di due distinte tipologie del cortigiano, addetta, l'una, al governo pubblico dello stato, l'altra alla persona privata del principe:

Questi sono due modi e, per così dire, due strade per le quali si perviene quasi egualmente a la grazia del principe: ma l'una è propria de i consiglieri e de' secretari, l'altra di compagni e di quelli che servono a la persona; e se questi per quella o quelli per questa caminassero, non ci giungerebbono così agevolmente (\$ 43$)$.

A questo punto il dialogo ha una nuova svolta argomentativa, introdotta dalla clausola del Forestiero Napolitano, che richiama uno dei principi fondamentali della paideia classica, che nella scelta del percorso formativo dell'adolescente chiede che si tenga conto sia del suo ingenium sia di quanto sia conveniente al proprio status (e questo è norma primaria dell'etica classica e classicistica): «Ciascun dunque deve elegger quella via che più gli si conviene, avendo risguardo a la nobiltà, a la ricchezza, a l'industria, al

\footnotetext{
${ }^{24}$ L'istanza dell'occultamento e del nascondersi, come quella dell'apparire saranno poi declinate nel Malpiglio come pratiche di un generale fingere e simulare sulla scena della corte: ma di questo più avanti.
} 
valore e a l'altre condizioni datele da la natura e da la fortuna». Malpigli conferma di avere fatto buoni studi riconoscendo nelle parole del Forestiero Napolitano il riferimento al gnōthi sautón iscritto sul tempio di Apollo a Delfi, e questa «cognizion di se stesso dee preceder tutte l'altre» (\$44).

Il cortigiano che sappia diventare amatore della persona del principe, e ci riesca a esserlo, provocherà inevitabilmente l'invidia degli altri cortigiani, e per schivarla (ma anche per schivare l'invidia del principe verso il cortigiano troppo virtuoso) gli converrà praticare quell'occultamento di cui i due hanno prima ragionato, perché solo «occultando il cortigiano schiva la noia del principe, e occultando ancora par ch'egli possa celarsi da l'invidia cortigiana» (\$ 47). Ma non bastano tutte le arti disponibili (cioè, le varie competenze nelle «scienze divine e umane», nonché nella «istoria [...] poesia e [...] arte oratoria») a evitarla, e perciò «in questo è la vita de' cortigiani» infelice. La pratica dell'occultamento deve pertanto realizzarsi come pratica della virtù della prudenza, che «supera ne le corti tutte le difficultà o la cognizione de le cose naturali» ed è non solo «la principal virtù de le corti» (\$50), ma anche quella che guida le virtù dei costumi, cioè dei comportamenti ordinari (in quanto habitus), dei quali «è lume e guida e quasi imperatrice» $(\mathbb{5 1})^{25}$.

Anche se l'argomentazione del dialogo, nel suo tentativo di registrare tutte le caratteristiche della Corte contemporanea è molto veloce ed è fatto più di enunciazioni che di elaborazioni, riesce purtuttavia a cogliere gli aspetti nuovi del servire a Corte e delle relazioni cortigiane proprio con la secca indicazione dell'assoluto primato della virtù prudenza, una virtù che ha subito una profonda mutazione semantica: da virtù cardinale del discernimento, cioè della discrezione (nel senso di allora), che nell'etica classica e classicistica (aristotelica e tomistica) consiste nel saper distinguere ciò che è giusto e buono secondo convenienza e differenza: questa saggezza pratica funzionale al conveniente agire nelle diverse situazioni e occasioni, acquista ora una nuova dimensione, come primaria istanza di cautela per evitare ogni pericolo (nel senso, cioè, che è pervenuto fino al nostro lessico quotidiano), come strumento di sopravvivenza. Il Malpiglio è dunque un indicatore sensibilissimo dei processi in corso nelle culture (in senso antropologico) delle società di Corte e ne registra quelle tendenze che ne connoteranno le dinamiche relazionali nell'età barocca, e oltre, sia nel rapporto tra principe e cortigiani (con le nuove distinzioni di ruoli e funzioni) sia tra i cortigiani. Anche questo particolare aspetto del discorso tassiano sulla Corte riprende l'impostazione del Cortegiano, sempre molto consapevole delle mutazioni che lo

25 Tasso impiega una similitudine per argomentare il variegato rapporto della prudenza con le virtù (di cui è «quasi imperatrice», e le nomina: «[...] sì come ne le pitture con l’ombre s'accennano alcune parti lontane, altre sono da' colori più vivamente espresse, così avverrà parimente de le virtù che sono con la prudenza: percioché la fortezza e la magnanimità e alcun'altre si veggono adombrate e paiono quasi di lontano discoprirsi; ma la magnificenza, la liberalità e quella che si chiama cortesia con proprio nome e la modestia è dipinta con più fini colori ch'abbia l'artificio del cortigiano, anzi viva più tosto: parimente le virtù del conversare, io dico la verità, l'affabilità e la piacevolezza» [\ 52]); anche per ciascuna di queste virtù nominate sarebbe possibile illustrare (e lo si dovrebbe fare) le mutazioni semantiche intervenute nei loro modi di definizione e d'uso nei discorsi di fine secolo pertinenti all'etica (in quelli dei moralisti non di professione, però: cioè, non in quelli di filosofi e teologi), mutazioni che anticipano o orientano le mutazioni del secolo successivo rispetto alla tradizione aristotelica e tomistica. 
scorrere del tempo produce nelle società e nelle culture (lingua compresa) degli uomini, $\mathrm{e}$ in particolare nelle corti, ma consapevole anche della loro grande varietà nella sincronia.

L'argomentazione primaria del dialogo è a questo punto conclusa, e infatti Malpigli torna a citare Castiglione non solo nominandolo, ma utilizzando la stessa parola che nel Cortegiano autoconnota il libro stesso «come un ritratto di pittura della corte d'Urbino» (Dedica 14): «Io veggio non solo il disegno, ma l'imagine del cortigiano e 'l ritratto già colorito. E se l'altro del Castiglione fu per quella età ne la qual fu scritto, assai caro dovrà essere il vostro in questi tempi [...]». Malpigli dimostra anche di avere compreso la lezione del Forestiero Napolitano e infatti conclude riassumendo tutto il percorso argomentativo fin qui compiuto in una sola icastica battuta che sinteticamente rappresenta il senso della nuova prudenza cortigiana: quando si riferisce a «questi tempi», a tempi «in cui l'infinger è una de le maggior virtù» $(\$ 53)^{26}$.

Da questo approdo, che di per sé potrebbe essere conclusivo, in poi il dialogo aggiunge diversi altri temi, tutti brevemente trattati, più che altro per completare con altri dettagli lo sfondo del "ritratto" della Corte e del cortigiano che vi presta servizio. Così è per la necessaria mediocrità (pur sempre aurea per classica e classicistica definizione) nelle pratiche della prudenza come virtù dell'infingere e del simulare; così è per l'altrettanto necessario saper «adattar le cose antiche a' tempi nostri» (\$55), purché sia fatto acconciamente; e ancora: rifuggire le dispute e le contenzioni intraprese tanto per sembrare bravo dialettico, perché sono «cosa odiosetta anzi che no», mentre saranno invece apprezzati come «assai piacevoli [...] que' contrasti d'ingegno che son convenienti a' cortegiani» (\$56): e il modello è ancora quello dei dialoghi di Castiglione.

Che la trattazione del tema della corte sia conclusa lo dimostra infine la ripresa per opera del Forestiero Napolitano di quella definizione da cui il dialogo aveva preso le mosse («la corte è una congregazione d'uomini raccolta per onore» [ [57]), da una nuova angolazione, però. Malpigli è incalzato da una serie di domande: tale congregazione è «perfetta o imperfetta»? e dal momento che la risposta non può che essere positiva «è bastevole a sé stessa?», cioè «contiene in sé stessa tutto ciò che l'è necessario?» (\ 58); e dal momento che, dice Malpiglio, la corte ha in sé «a bastanza» quanto occorre per le sue necessità, il Forestiero Napolitano lo incalza: «Ma tutte l'arti che son necessarie a la vita civile son parimente necessarie al cortigiano?» (\$58). Anche a questa domanda la risposta è obbligata, ma porta a quello snodo argomentativo al quale l'istitutore, il Forestiero Napolitano, voleva maieuticamente far giungere il suo giovane allievo, sollecitandolo a entrare nel merito delle arti necessarie: sono anche quelle che «si ricercano per ornamento, come son la pittura e la scoltura», e «anzi forse [lo sono] tanto più quanto, essendo la corte più risguardevole, deve abondar di più nobili ornamenti?» ( $(\mathbb{5} 59)$.

Il questo modo il dialogo sembra citare, minutamente dettagliandola e soprattutto rielaborandola, la famosa definizione del Palazzo ducale di Urbino che

${ }^{26}$ Questa licenza d'infingere nelle pratiche pertinenti all'etica dovrebbe essere opportunamente correlata alla «licenza di fingere», fondamentale per il poeta moderno, di cui Tasso tratta nei Discorsi dell'arte poetica e in altre sue opere. 
«non un palazzo, ma una città in forma de palazzo esser pareva» (I, 2, 5), ma più che Urbino sembra prospettare Versailles: nella corte del Malpiglio, ci sono infatti «tutti gli artefici», anche quelli che sono parte della città, cosicché «il sartore sarà non solamente sartore ma cortigiano, e 'l calzolaio e l'orafo e "l pittore e lo scultore e ciascun altro» ( $\mathbb{S}$ 60), e per il loro essere tutti cortigiani sono anche i «più eccelenti» (\$ 60). La conclusione di questo segmento argomentativo supplementare è pertanto prevedibile: «La corte dunque è una raccolta di tutte l'eccelenze di tutte l'arti e tutte l'opere le quali sono fatture [prodotti del fare]: laonde parte de' cortigiani a contemplare, parte a l'operare, parte al fare saranno intenti» ( $\left.\int 61\right)$; compresi anche i poeti, gli oratori, i musici, gli scienziati, i matematici: tutti «son in quel modo cortigiani che son cittadini» ( $(52)$.

L'obiettivo del Forestiero Napolitano non è però giungere a questa conclusione, bensì tornare a ragionare (tutto ciò doverosamente premesso) a quanto pertiene propriamente al cortigiano, ancora in termini distintivi rispetto al cittadino:

[...] propriamente cortigiano è colui ch'attende a l'azione e al negozio: e questo è il prudente al quale ne le corti s'appertiene il commandare intorno a tutte l'arti e tutte le scienze non altramente che faccia l'uom civile ne la città (\$ 62).

Non è soltanto il Palazzo-Città di Urbino a svanire, ormai remoto come modello socioculturale, ma anche, almeno in questa sua occorrenza, il valore semantico di civile che la tradizione umanistica (e in particolare Erasmo da Rotterdam) aveva trasferito dalla sua originaria pertinenza "civica" a una nuova pertinenza universale, in quanto elemento costitutivo e proprio di una civilitas culturale e morale. Questo impiego tassiano di civile potrebbe essere un minimo segno, comunque significativo, di quella consapevolezza della propria distinzione identitaria, sociale e culturale, che già separa e sempre più separerà le società aristocratiche cortigiane d'Antico regime, in quanto universi autosufficienti e autoreferenti ${ }^{27}$.

A questo punto il Forestiero Napolitano propone un'osservazione che risulta estranea al contesto argomentativo del dialogo e sembra riferirsi alla condizione personale di Tasso «nato ed allevato in corte» e di fatto cortigiano di tante corti, ma sempre con uno statuto che non è propriamente quello di un cortigiano, o almeno vorrebbe che non lo fosse o che almeno non fosse considerato come tale (quale, a esempio, era la condizione di suo padre). L'osservazione è questa:

Color dunque che son volti a la contemplazione de le cose grandi e sublimi, tutto che non siano cortigiani propriamente, tanto dovrebbono esser partecipi de la prudenza e de le maniere laudevoli de la corte, quanto bastasse a farli più cari al principe e a ciascun altro (\$63).

27 Sarebbe opportuna un'analisi, che qui non posso fare, dei numerosi impieghi della famiglia semantica di civile in Tasso e in particolare del sintagma «uomo civile», che spesso è usato in termini ancora distintivi rispetto a cortigiano, ma anche a ignobili. 
Per quanto speciale, persino unico, possa essere lo statuto del loro stare in Corte, coloro che contemplano «cose grandi e sublimi», come i massimi poeti (e Tasso sa di esserlo), devono comportarsi come tutti gli altri cortigiani, secondo prudenza soprattutto, e imitare le «maniere laudevoli de la corte» (e questo è il problema drammatico che devasta l'esperienza cortigiana di Tasso a Ferrara e lo porta alla reclusione in Sant'Anna). Non soltanto i poeti e gli altri contemplativi di «cose grandi e sublimi», ma pure tutti coloro che «esercitano l'arti», anche quelle ignobili, e che entrano in rapporto con la corte: secondo il Forestiero Napolitano devono essere partecipi della «prudenza de' superiori», perché in questo modo «prendono qualità e gentilezza da la corte» (\$ 64).

L'ultimo spunto argomentativo del dialogo riguarda il riconoscimento di un'altra peculiarità della corte, che i Malpigli conoscono bene, dal momento che sono lucchesi alla corte di Ferrara: quella di essere un «adunanza di varie nazioni» (nel senso di allora: persone diverse per luogo di nascita),

le quali non usano una lingua solamente, ma con gli Italiani sono mescolati i Tedeschi, i Francesi, i Boemi, i Greci e quelli d'altre provincie, fra' quali è gran concordia nel servire al principe: e s'alcuna contesa è in questo, è contesa di gentilezza e di cortesia (\$ 65).

Questo riferimento serve a chiudere il dialogo: la condizione del giovane Malpiglio che è arrivato (come già suo padre) da «lontane parti» serve a introdurre un'ultima similitudine, molto elaborata:

[...] sì come il sol nascente e l'altre stelle matutine paiono aggrandirsi per la copia de' vapori, così per lo favore acquistato ne l'età giovenile sogliono essere in pregio maggiore, sì veramente che 'l valore o la diligenza porga occasione al favore (\$ 67).

La giovane età di Giovanlorenzo Malpigli che entra a corte potrà essere per lui un vantaggio, se saprà far tesoro degli avvertimenti (o ricordi: nel senso di allora) dispensatigli dal Forestiero Napolitano, che ragionando su questo dato anagrafico riprende il topos delle età dell'uomo, evocando la condizione prima del vecchio cortigiano (stimato «per la riverenza» che suscita e quanto la sua età comporta in termini di saggezza e di prudenza), e poi del cortigiano che ha «l'età interposita» fra l'una (la gioventù) e l'altra (la vecchiaia), e non credo che ci sia bisogno di ricordare quanto fosse sviluppata nel Cortegiano la discussione sui vecchi e sui giovani: nel sistema della Corte si è valutati per quello che si fa e si dice e per come lo si fa e si dice (cioè, per la qualità delle sue diverse operazioni), e per questo la sua attività è «forse più sottoposta a l'invidia». Questo basta per il monito finale: «[...] però debbiam ricordarci di tutte quelle cose le quali sono atte a schivarla» (\$ 68).

Nelle battute conclusive del dialogo riprende la parola Vincenzo Malpigli, per ricordare che suo figlio è ancora troppo giovane per pensare al suo futuro e che «ora dee pensare più a lo studio ch'a la corte: nondimeno questi ragionamenti li saranno stati in vece di studio, perché molte cose può avere apprese, ch'egli non sapeva» ( $\mathbb{S}$ 
69) ${ }^{28}$. L'ultima parola spetta però doverosamente a chi ha condotto il dialogo dispensando il suo sapere, al Forestiero Napolitano, che ripropone la metafora dello sprone, con un estremo avvertimento: se apprendere le scienž è preliminare a qualsiasi scelta di vita, scegliendo la corte il giovane Malpigli dovrà «servirsene in quella guisa che si conviene a gentiluomo di corte»: perché in questo contesto «non è tanto necessaria la eccelenza de le lettere, quanto la prudenza e l'accortezza di saperle a tempo manifestare», ma «nondimeno l'una senza l'altra pare imperfetta».

\footnotetext{
${ }^{28}$ Ricordo che proprio in questo consiste l'elogio della conversazione secondo Stefano Guazzo: «[...] giova più al letterato un'ora ch'egli dispensi nel discorrere con suoi eguali, ch'un giorno di studio in solitudine» (cito da La civil conversażione, ed. Amedeo Quondam [Modena: Franco Cosimo Panini, 1993], $32,1 \mathrm{~A} 18 \mathrm{n}$ ), e tanto più questo assioma vale per un giovane discepolo che abbia passato un'ora ad ascoltare la lezione di un maestro come il Forestiero Napolitano.
} 


\section{BIBLIOGRAFIA}

Bongi, Salvatore, Storia di Lucrezia Buonvisi lucchese raccontata sui documenti (Lucca: B. Canovetti, 1864; Lucca: Pacini Fazzi, 1978, ristampa anastatica).

Bozzola, Sergio, “'Questo quasi arringo del ragionare'. La tecnica dei Dialoghi tassiani”, Italianistica 26, 2 (1997): 253-278.

Castiglione, Baldassare, Il Libro del Cortegiano, ed. Amedeo Quondam (Roma: Bulzoni Editore, 2016).

Cattini, Marco, e Marzio A. Romani, "Le corti parallele: per una tipologia delle corti padane dal XIII al XVI secolo", in La Corte e lo spario: Ferrara estense, ed. Giuseppe Papagno e Amedeo Quondam (Roma: Bulzoni Editore, 1982), 47-82.

Chiarelli, Angelo, "'Questa concordia è sempre ne le cose vere'. Note per una contestualizzazione de Il Costante overo de la clemenza di Tasso", Filologia \& Critica 16 (2016): 257-270, https://doi.org/10.1400/266543.

—, "Una 'congregazione di uomini raccolti per onore'. Tentativi di aggiornamento della teoria cortigiana nella dialogistica e nella prosa tassiana", Rassegna della letteratura italiana 121, 1 (2017): 34-43.

Cox, Virginia, "Tasso's Malpiglio overo de la Corte: The Courtier Revisited", The Modern Language Review 90, 4 (1995): 897-918, https://doi.org/10.2307/3733065.

Dionisotti, Carlo, Geografia e storia della letteratura italiana (Torino: Einaudi, 1967).

Fedele, Dante, "Uno scritto sull'ambasciatore del secondo Cinquecento: Il Messaggiero di Torquato Tasso", Il Pensiero Politico 51 (2018): 113-125.

Ferro, Giovanni, Teatro d'imprese (Venezia: Giacomo Sarzina, 1623).

Girardi, Maria Teresa, “Le lettere non 'poetiche' di Tasso come luogo di riflessione poetica", in Ricerche sulle lettere di Torquato Tasso, ed. Clizia Carminati ed Emilio Russo (Sarnico: Edizioni di Archilet, 2016), 25-43.

Guazzo, Stefano, La civil conversazione, ed. Amedeo Quondam (Modena: Franco Cosimo Panini, 1993).

Lucarelli, Massimo, "Il nuovo Libro del Cortegiano: una lettura del 'Malpiglio' di Tasso", Studi Tassiani 52 (2004): 9-24. 
Papi, Fiammetta, "Sulla semantica della cortesia. Riflessioni su una definizione dantesca", Italianistica. Rivista di letteratura italiana 44, 2 (2015): 209-221, https://doi.org/1 $\underline{0.1400 / 235710 .}$.

Pignatti, Franco, "I Dialoghi di Torquato Tasso e la morfologia del dialogo cortigiano rinascimentale", Studi Tassiani 36 (1988): 7-43.

Quondam, Amedeo, Forma del vivere. L'etica del gentiluomo e i moralisti italiani (Bologna: Il Mulino, 2010).

-, "Giochi di corte", in Giocare tra Medioevo ed età moderna. Modelli etici ed estetici per l'Europa, ed. Francesca Aceto e Francesco Lucioli (Roma: Fondazione Benetton Studi Ricerche-Viella, 2019), 87-112.

-, "La gloria del cavallo", in Movilidad cortesana y distinción: coches, tiros y caballos. II Congreso Internacional "Las caballerizas reales y el mundo del caballo", ed. Juan Aranda Doncel y José Martínez Millán (Córdoba: IULCE-Córdoba Ecuestre, 2019), 13-44.

Raimondi, Ezio, "La prigione della letteratura", Introduzione a Torquato Tasso, Dialoghi, ed. Giovanni Baffetti (Milano: Rizzoli, 1998), 9-56.

Residori, Matteo, “'Del fuggir la moltitudine'. Néoplatonisme et scepticisme dans le Malpiglio secondo du Tasse", Italique. Poésie italienne de la Renaissance 5 (2002): 95108, https://doi.org/10.4000/italique.150.

Russo, Emilio, "I dialoghi tassiani e la cultura di fine Cinquecento", in L'ordine, la fantasia e l'arte. Ricerche per un quinquennio tassiano (1588-1592) (Roma: Bulzoni Editore, 2002), 9-67.

Schiera, Pierangelo, Specchi della politica. Disciplina, melancolia, socialità nell'Occidente moderno (Bologna: Il Mulino, 1999).

Sodini, Carla, "Lucrezia Malpigli", in Dizionario Biografico degli Italiani, LXVIII (Roma: Istituto della Enciclopedia Italiana, 2007), ad vocem. Disponibile in www.treccani.it [data di consultazione: 10/2/2021].

Tasso, Torquato, Le lettere disposte per ordine di tempo, ed. Cesare Guasti, 5 voll. (Firenze: Le Monnier, 1852-1855), I, II, III y V.

—, Dialoghi, ed. critica Ezio Raimondi (Firenze: Sansoni, 1958).

—, Dialoghi: Il Messaggiero, Il padre di famiglia, Il Malpiglio, La Cavalletta, Il Molra, ed. Bruno Basile (Milano: Ugo Mursia Editore, 1991). 
Vagni, Giacomo, "Fra realtà biografica e verosimile letterario. Primi appunti sui personaggi dei Dialoghi di Tasso", in "Imitazione di ragionamento". Saggi sulla forma dialogica dal Quattro al Novecento, ed. Vincenzo Caputo (Milano: Franco Angeli, 2019), 127-138.

-, "Oltre l'autocensura. Note sul rifacimento del dialogo tassiano Il Nifo overo del piacere", Rivista di letteratura italiana 37 (2019): 39-56, https://doi.org/10.19272 $\angle 201902203002$.

Recibido: 28 de febrero de 2021

Aprobado: 25 de mayo de 2021 